\title{
A Quantitative Image Analysis for the Cellular Cytoskeleton during In vitro Tumor Growth
}

Mohammad A Al-Mamun ${ }^{\mathrm{a}, *}$, Worawut Srisukkham ${ }^{\mathrm{b}}$, Dewan Md. Farid ${ }^{\mathrm{c}}$, Lorna Ravenhill ${ }^{\mathrm{d}}$, Li Zhang ${ }^{\mathrm{b}}$, Alamgir Hossain ${ }^{\mathrm{e}}$ and Rosemary Bass ${ }^{\mathrm{f}}$

${ }^{\text {a }}$ Department of Population Medicine \& Diagnostic Sciences, College of Veterinary Medicine, Cornell University, Ithaca, NY 14850, USA

${ }^{\mathrm{b}}$ Computational Intelligence Group, Faculty of Engineering \& Environment, Northumbria University, NE1 8ST, UK

${ }^{\mathrm{c}}$ Department of Computer Science \& Engineering, United International University, Dhaka 1209, Bangladesh

${ }^{\mathrm{d}}$ School of Biological Sciences, University of East Anglia, Norwich, Norfolk, NR4 7TJ, UK

${ }^{\mathrm{e}}$ Anglia Ruskin IT Research Institute (ARITI), Anglia Ruskin University, Cambridge, CB1 1PT, UK

${ }^{\mathrm{f}}$ University of East Anglia, Norwich Research Park, Norwich, NR4 7TJ, UK

\begin{abstract}
The cellular cytoskeleton is a dynamic subcellular structure that can be a marker of key biological phenomena including cell division, organelle movement, shape changes and locomotion during the avascular tumor phase. Little attention is paid to quantify changes in the cytoskeleton while nuclei and cytoplasmic both are present in subcellular microscopic images. In this paper, we proposed a quantitative image analysis method to analyze subcellular cytoskeletal changes using a texture analysis method preceded by segmentation of nuclei, cytoplasm and ruffling regions (area except nuclei and cytoplasm). To test and validate this model we hypothesized that Mammary Serine Protease Inhibitor (maspin) acts as cytoskeleton regulator that mediates cell-extracellular matrix (ECM) adhesion in tumor. Maspin-a tumor suppressor gene shows multiple tumor suppressive properties such as increasing tumor cell apoptosis and reducing migration, proliferation, invasion, and overall tumor metastasis. The proposed method obtained separated ruffling regions from segmentation steps and then adopted gray-level histograms (GLH) and grey-level co-occurrence matrix (GLCM) texture analysis techniques. In order to verify the reliability, the proposed texture analysis method was used to compare the control and maspin expressing cells grown on different ECM components: plastic, collagen I, fibronectin and laminin. The results show that the texture parameters extracted reflect the different cytoskeletal changes. These changes indicate that maspin acts as a regulator of the cell-ECM enhancement process, while it reduces the cell migration. Overall, this paper not only presents a quantitative image analysis approach to analyze subcellular cytoskeletal architectures but also provides a comprehensive approximation on how maspin regulates cell-ECM interactions to show potential tumor suppressive properties.
\end{abstract}

Keywords: Cellular cytoskeleton, MASPIN, Tumor growth, Quantitative image analysis, Markercontrolled watershed segmentation, Texture analysis.

*Corresponding author:

Email addresses: ma875@cornell.edu (Mohammad A Al-Mamun),

srisukkham.worawut@northumbria.ac.uk (Worawut Srisukkham), dewanfarid@cse.uiu.ac.bd (Dewan Md.

Farid), lorna.Ravenhill@rhul.ac.uk (Lorna Ravenhill), li.zhang@northumbria.ac.uk (Li Zhang),

alamgir.hossain@anglia.ac.uk (Alamgir Hossain), r.bass@uea.ac.uk (Rosemary Bass) 


\section{Introduction}

The cellular cytoskeleton is a highly dynamic structure that maintains cell shape and itself gets remodeled constantly in a living cell (Ridley 2011). It is required during cell division, organelle movement, and cellular motion. It provides protection, shape and elasticity to the cell contents spanning the cytoplasm. In tumors, the cellular cytoskeleton aids all kinds of cellular morphogenesis including migration and adhesion required for a cell to extend, retract, and stabilize. The cytoskeleton aids cell locomotion during the avascular phase of tumor growth (Chakrabarti et al., 2015). At the leading edge of a motile cell, focal contacts hook the cytoskeleton to extracellular matrix (ECM) for providing a net force of forward traction. Then, the trailing edge disassembles its adhesive contacts and allows retraction to commence by actomyosin contraction (Sander et al., 1999). Novel imaging techniques (e.g. fluorescent microscopy, confocal and optical coherence tomography etc.) have provided a detailed view of cellular and molecular migration dynamics in cancer cells during in vitro and in vivo study (Matuszewski et al., 2011).

Essentially, changes in the cellular cytoskeleton can be crucial markers of cell migration and adhesion when Mammary Serine Protease Inhibitor (maspin), a tumor suppressor gene, is present in the tumor cells. Previously, maspin was discovered in an in vivo study where it was silenced in breast cancer (Zou et al., 1994). After this, maspin was characterized as a tumor suppressor because of its involvement in three key areas of tumor development: metastasis, apoptosis, and angiogenesis. In particular, researchers studied its localization (Zou et al., 1994), regulation of expression (Cella et al., 2006), and more recently protein interactions of maspin (Endsley et al., 2011). Many studies suggest that maspin is present at the cell surface (Sheng et al., 1996; Pemberton, et al., 1997; Seftor et al., 1998; Ngamkitidechakul, et al., 2001; 2003; Law et al., 2005 Cella et al., 2006; Khalkhali-Ellis and Hendrix, 2007; Bass et al., 2009; Ravenhill et al., 2010; Endsley et al., 2011). But, exogenous activities of maspin are still debatable (Teoh et al., 2010; 2014). To date, few computational attempts have been taken to investigate the fact that maspin resides exogenously and acts as cytoskeleton regulator by reducing cell migration and increasing cell-ECM adhesion (Al-Mamun et al., 2013; 2016a). Recently, Al-Mamun et al., (2016b) presented a multidisciplinary quantitative image model where reduction of cell migration phenomena was investigated through both in vitro and computational study. This study measured the nuclei, cytoplasmic and ruffling regions using different quantitative descriptors like area, perimeter, and circularity of the cells, fractal dimension, and integrated optical density. But no texture analysis method was used to detect changes in the periphery at the cell membrane. Recently, some studies attempted quantitative analysis of subcellular structures of a cell such as grayscale analysis of microtubules of cardiac myocytes (Dang et al., 2015), combined method of gray-level-histogram (GLHs), a gray-level co-occurrence matrix (GLCM) (Lu et al., 2014) and wavelet packet energy analysis (WPEA) to analyze different facets of microtubules morphology 
(Lan et al., 2015). These motivated us to hypothesize that cellular cytoskeletal changes due to maspin can be detectable by quantifying the textures of ruffling regions at the surface of cell membrane.

Identifying cellular changes from confocal microscopic images is a challenging task and usually, it requires experienced assessors to be interpreted. Presently, having a robust system that allows rapid analysis of large numbers of images would be of value. Practically, most subcellular microscopic images do not just contain single cells, but also clumped and layered cells. For analyzing a complex subcellular microscopic image we need to define areas containing the nuclei, cytoplasm, and peripheral substances. Generally, digital confocal microscopy images need a good segmentation method to allow cells to be segmented even if they have grown in clumps or in a layered fashion. Many researchers have developed different image processing methods to quantify the endo- and exogenous structures of a cell. Usually, segmentation of nuclei and/or cytoplasm is performed as the first step and then other quantification techniques are implemented on those segmented pieces. Some popular segmentation techniques include Otsu's method (Otsu, 1979), edge detection (Wählby et al., 2004), watershed (Mukherjee et al., 2004), mean shift method (Debeir et al., 2005), level set algorithms (Nath et al., 2006), active contour models (Kayser et al., 2009), sliding band filter (Quelhas et al., 2010), and iterative thresholding (Cai et al., 2014). To overcome the limitation of over-segmentation of the watershed method, marker-controlled watershed techniques have been developed to improve the performance (Yang et al., 2006; Fenistein et al., 2008; Plissiti et al., 2011, Koyuncu et al., 2012; Arco et al., 2015). Before applying a segmentation method, morphological analysis is helpful to extract useful information from the cellular images (Amini et al., 2010; Plissiti et al., 2011). A two-step binarization method is proposed to split the clumped nuclei (LaTorre et al., 2013).

Extracting quantitative texture features from microscopic images of the cellular cytoskeleton warrants proper segmentation of cell parts - nuclei, cytoplasm and other regions. Though selecting an appropriate segmentation method depends on three considerations: a) whether the method will be able to separate the features of interest when there is great variability in background patterns and intensity in images, b). whether it will be able to resolve the overlapping nuclei or cell separation problem and c) whether it will require human intervention and supervision to solve the problem. Sometimes, segmentation becomes more difficult because of different heterogeneous complexity in the image samples. Firstly, inherent image acquisition processes may generate noisy image samples due to the dust on the confocal slits in the microscope. The noisy image samples must be pre-processed before supplying to the segmentation algorithm, otherwise, these may create a problem during binarization process (LaTorre et al., 2013). Secondly, overlapped nuclei may generate over-segmentation, because the clumped nuclei or cytoplasm may belong to different cells. Thirdly, even though the nuclei splitting algorithm successfully segment the nuclei, but the division of clumped cytoplasm area may arise the under or over-segmentation problem (Lindblad et al., 2004). Finally, even both nuclei and 
cytoplasm segmentation algorithms may successfully segment the major cell parts; but they may not distinguish the outer cellular parts (cytoskeleton). In these cases, co-staining of nuclei and cytoplasm during in vitro experiment setup may resolve some problems by providing separate nuclei and cytoplasm image samples for analysis (Lindblad et al., 2004). In this paper, we used co-stained image samples of nuclei and cytoplasm for four ECM substrates, fibronectin, laminin, collagen I and plastic. Here, we propose a quantitative texture analysis method to analyze the cellular cytoskeletal morphology for two groups of image samples: control and maspin for four ECM components. A marker-controlled watershed segmentation method was used to segment two channel image samples into three cellular parts: nuclei, cytoplasm and ruffling area. Then we used several texture analysis methods to analyze the texture of ruffling regions. We also perform comparison and validation of the method with an existing method using domain expert's knowledge.

The rest of the paper is organized as follows. Section 2 presents the details of the in vitro, biochemical fractionation, western blotting, image acquisition technique, segmentation model, and texture analysis methods. Section 3 presents the segmentation and texture analysis results. It also provides the in vitro supports to the texture analysis finding using biochemical fractionation of F and G-actin. Section 4 discusses the relevance of the results with the biological hypothesis and contribution and implications. Section 5 summarizes the paper and states future direction of the proposed method.

\section{Materials and Methods}

\subsection{In vitro methods}

MCF-7 cell lines with differing maspin expression were generated and maintained as before (Ravenhill et al. 2010); "control cells" are maspin null MCF-7-pcDNA3.2, "maspin cells" are MCF7-pcDNA3.2-maspin transfected cells expressing maspin. Cell lines were authenticated by the DNA Diagnostics Centre (London, UK). All cell culture reagents, Alexa Fluor 568 labelled phalloidin and secondary antibodies were from Life Technologies (Paisley, UK). Commercially available mouse monoclonal antibodies were used to detect maspin (BD Biosciences, Oxford, UK). Control IgG was from Dako (Ely, UK).

\subsection{Image acquisition}

Coverslips were coated with isolated ECM components at $5 \mu \mathrm{g} / \mathrm{ml}$ for 15 hours at $4^{\circ} \mathrm{C}$. Cells were plated and incubated for 17 hours, then fixed as subconfluent monolayers with $4 \%$ formaldehyde and washed with PBS (phosphate buffered saline). For actin staining 0.2U of Alexa Fluor 568 labelled phalloidin was added to each sample and incubated in PBS for 40 minutes at room temperature. This was followed by two PBS washesSlides were mounted with hydromount (National Diagnostics, GA, USA). Cells were visualized under a Charge Coupled Device (CCD) upright microscope or an LSM 
confocal microscope (Carl Zeiss Ltd, Hertfordshire, UK). Images were captured with Axiovision 4.7.1 software and Zeiss LSM Examiner 4.0. The real image sample is shown in Figure 1; three interested regions (nuclei, cytoplasm, and ruffling region) are marked.

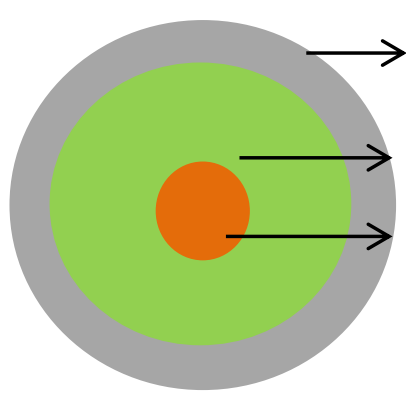

(a)

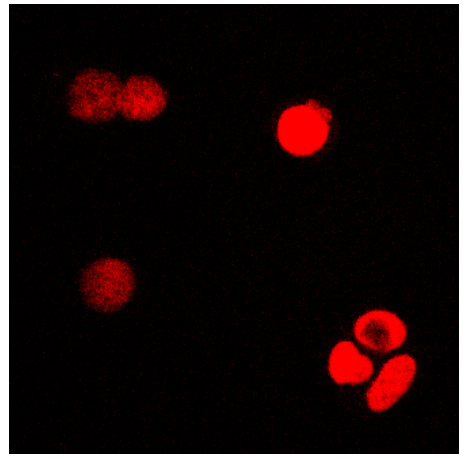

(b)

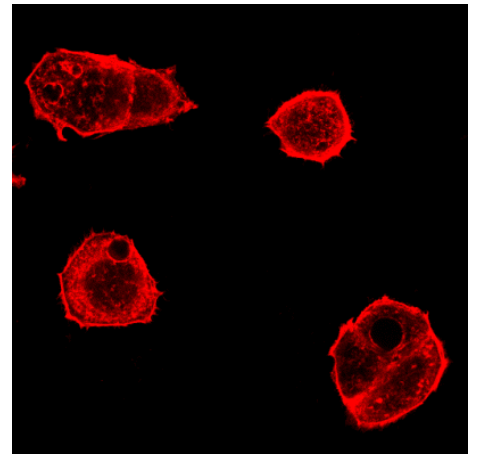

(c)

Figure 1. The grayscale two channel image samples extracted from LSM confocal microscope: a) three parts of a cell in a sketch b) channel 1: nuclei, and c) channel 2: cytoplasm with membrane.

\subsection{Biochemical fractionation}

The method for fractionating actin was based on that described elsewhere (Zou et al., 2007). Cells were plated onto the substrate at $2 \times 10^{5} / \mathrm{ml}$ in serum free medium and incubated for 17 hours. After 24 hours cells were washed twice in Stabilization Buffer (50mM PIPES pH 6.9, 50mM sodium chloride, 5mM EGTA, 5mM magnesium chloride, $1 \mathrm{mM}$ adenosine triphosphate, $1 \mathrm{mM}$ Dithiothreitol, $5 \%$ (v/v) glycerol, 0.1\% (v/v) $\beta$-mercaptoethanol). Extraction Buffer (Stabilization Buffer supplemented with $0.5 \%$ (v/v) Triton-X100 and Protease Inhibitor Cocktail (Roche, West Sussex, UK) was added for 10 minutes at $37^{\circ} \mathrm{C}$ and soluble proteins removed. The culture plate was then washed in Extraction Buffer and insoluble proteins scraped into protein sample buffer warmed to $37^{\circ} \mathrm{C}$.

\subsection{Cell lysates, SDS-PAGE and western blotting}

Cell lysates were prepared by the method reported previously (Ravenhill et al., 2010). For Western blotting, samples separated by SDS-PAGE on a $10 \%$ resolving gel were transferred to polyvinylidene difluoride membranes (Bio-Rad). Protein bands were detected by incubation with the appropriate antibody followed by HRP-conjugated secondary antibodies $(0.65 \mathrm{~g} / \mathrm{ml})$. For western blotting the concentration of primary antibodies is indicated in the figure legends, secondary antibodies were used at $1: 1000$.

\subsection{Segmentation model}

The segmentation model took two channel image samples (nuclei and whole cell) as input and then segmented the three cellular parts: nuclei, cytoplasm and ruffling region (the area without nuclei and 
cytoplasm). The proposed model is divided into three blocks: nuclei segmentation, cytoplasm segmentation and texture analysis. Figure 2 describes all the three blocks. Firstly, nuclei image was fed into the nuclei segmentation block where single and clumped nuclei were separated and segmented using watershed segmentation. Secondly, the separated seeded nuclei were used as marker for cytoplasm segmentation followed by a post-processing algorithm. Thirdly, bordered nuclei and cytoplasm were supplied to texture analysis block where ruffling area was extracted. Fourthly, ruffled regions were analyzed using GLHs and GLCM methods. Finally, the texture parameters were analyzed using statistical software packages.

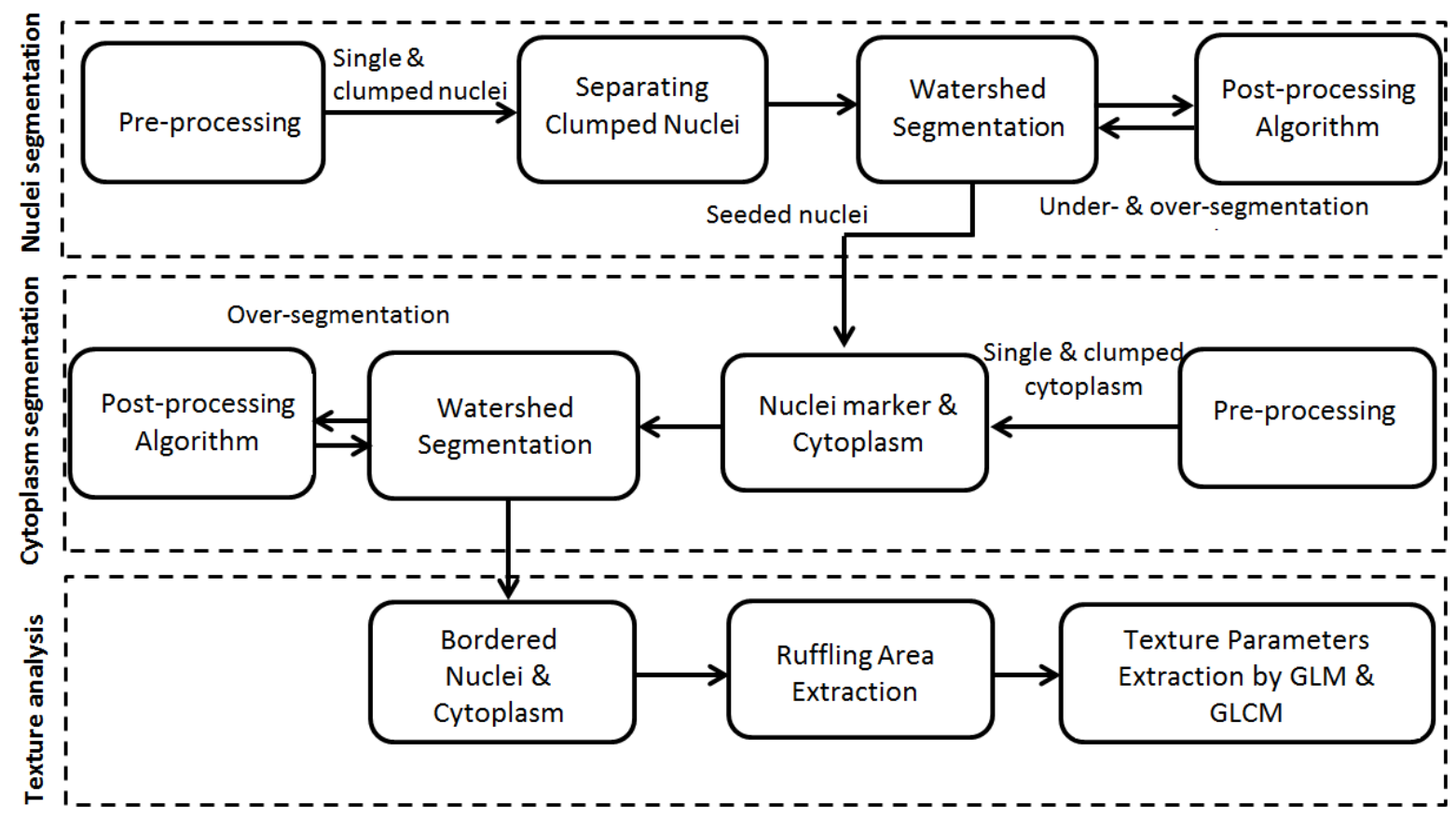

Figure 2. A general schematic of proposed quantitative image analysis method. Abbreviations: GLM: gray-level histogram, GLCM: gray-level co-occurrence matrix.

\subsection{Nuclei segmentation algorithm}

Each cell image comprised single and/or combined nuclei, cytoplasm and scattered ruffling area around cytoplasm. First, the model segmented the nuclei, as nuclei were used as a marker for cytoplasm segmentation using marker-controlled watershed segmentation technique (MCWS). A preprocessing algorithm was used to identify the cells from different background contrast. This preprocessing step is essential because some image samples had background contrast problem due to uneven illumination and striped pattern problems of the microscope. We used median filtering iteratively with contrast limited adaptive histogram Equalization (CLAHE) until the background problem resolved (Al-Mamun et al., 2016b). Normally, CLAHE is useful when both the background and foreground object are bright at the same time or else both are dark at the same time. During 
median filtering $3 \times 3$ neighbors and for CLAHE $8 \times 8$ tiles have chosen. Once we got the preprocessed image, we used the following procedure to segment the nuclei.

\section{Procedure 1: Nuclei Segmentation}

Step1: Filtering with sobel horizontal edge-emphasizing filter with gradient magnitude calculation for differential contrast between the foreground object bordered with the background (shown in Figure 3b)

Step 2: Morphological opening (object size- 50 pixels) to make a good nuclei seed (shown in Figure $3 c)$

Step 3: Two times dilation used to reconstruct the original nuclei size (shown in Figure 3d)

Step 4: Euclidian distance transform (EDT) has been used to create the separate line between clumped nuclei (Breu et al., 1995). During this process, h-minima level was set to 2 (shown in Figure 3e)

Step 5: Then clumped nuclei have been separated by applying watershed (shown in Figure 3f)

Step 6: If under- or over-segmentation occurred for any nuclei then it was sent to post-processing algorithm to solve the splitting problem

Step 7: Borders were drawn to delineate the segmented nuclei using 4 connecting neighborhood points (Srisukkham et al., 2013)

The details of the morphological operation and EDT are given in the supplementary file, section S1 and $\mathrm{S} 2$, respectively.

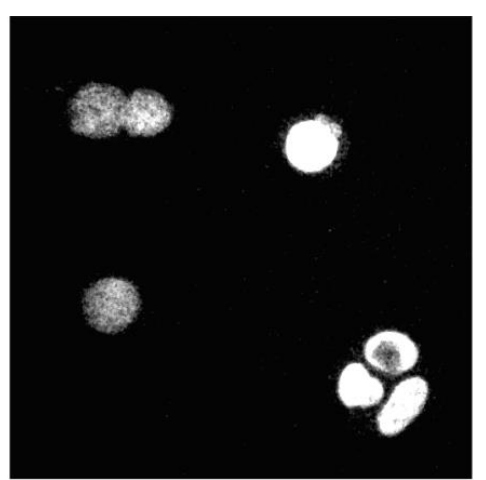

(a)

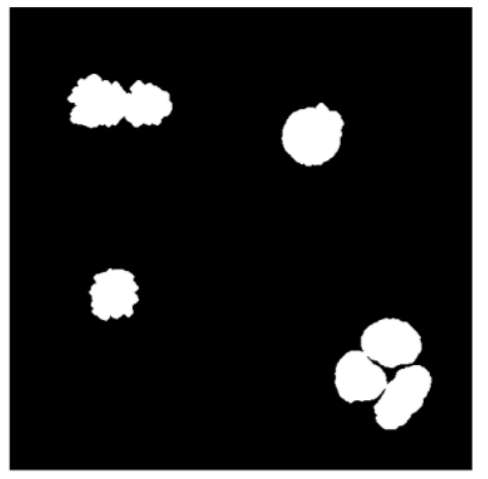

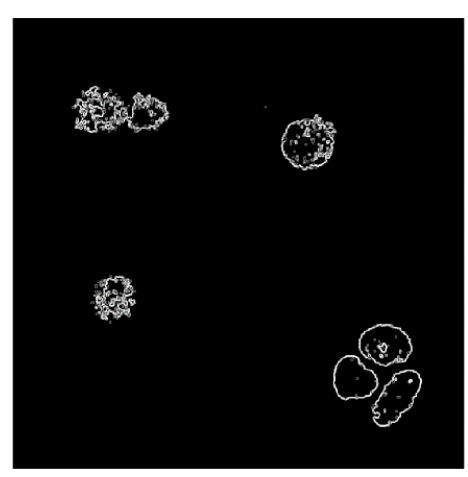

(b)

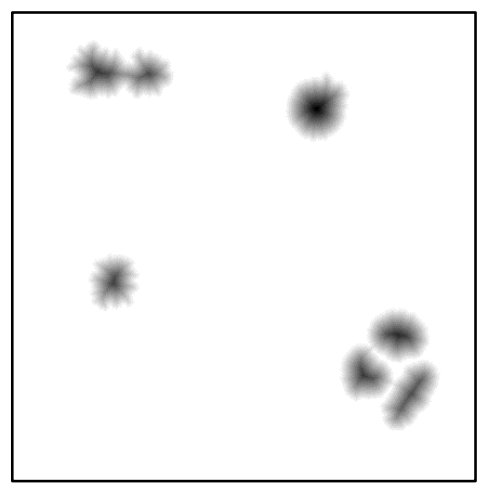

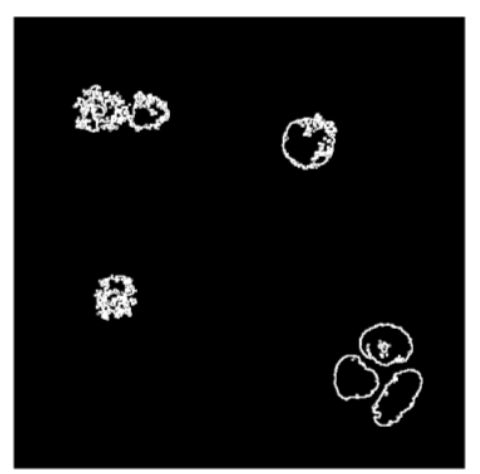

(c)

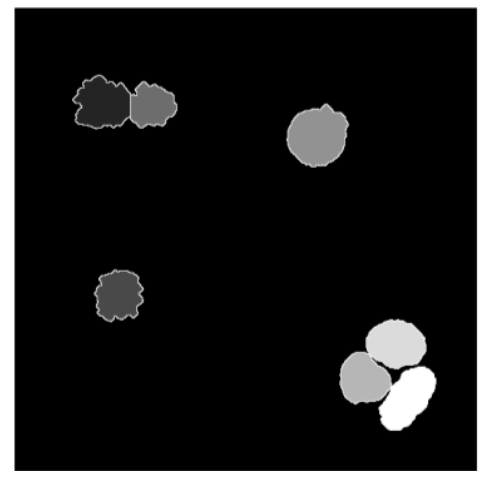


Figure 3. The steps of nuclei segmentation: (a) The preprocessed nuclei after preprocessing step, (b) the gradient magnitude variation of background and border, (c) after applying the morphological opening, (d) removing small objects size after 2 times dilation, (e) distance transform map of nuclei, (f) separated nuclei after applying watershed algorithms.

\subsection{Cytoplasm Segmentation}

The separated nuclei were used as seeds of cytoplasm segmentation. Before implementing the cytoplasm segmentation process, it was needed to run the preprocessing routine (described in section 2.6) for cytoplasm image samples. Once we obtained the preprocessed cytoplasm images, then we undertook the following procedure to segment the cytoplasm

\section{Procedure 2: Cytoplasm Segmentation}

Step1: Filtering with sobel horizontal edge-emphasizing filter with gradient magnitude calculation for

differential contrast between the foreground object bordered with the background (shown in Figure 4a)

Step 2: Morphological opening (object size-500pixels and line shaped-2pixels)

Step 3: Fill the holes of whole cytoplasmic area (shown in Figure 4b)

Step 4: Imposing the nuclei seed as a mask using extended- $h$-minima transform (regional minima of the h minima transform level) (shown in Figure 4c), The larger the h-value is, the fewer the numbers of the segmented regions. This step was crucial because once there was a good marker for the GWDT (shown in Figure 4d)

Step 5: Applying gray-weighted distance transform (GWDT) to the grayscale image and applied into -Inf background (shown in Figure 4e)

Step 6: Apply watershed to make the labelled cytoplasmic areas for single and combined cells (shown in Figure 4f)

Step 7: Borders were drawn to delineate the segmented nuclei using 4 connecting neighborhood points (Srisukkham et al., 2013)

The details of GWDT method is discussed in supplementary file in section S3. 


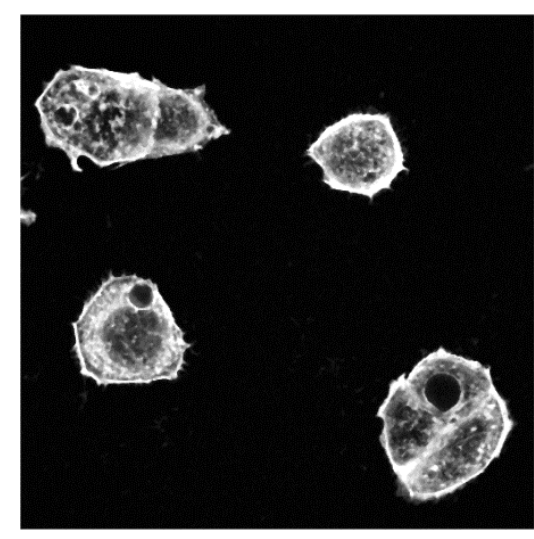

(a)

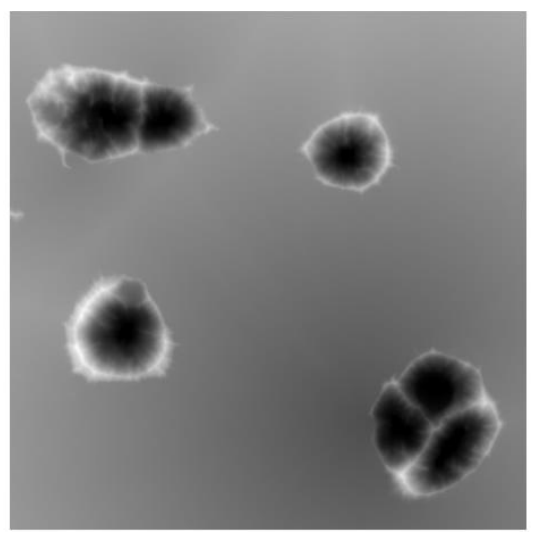

(d)

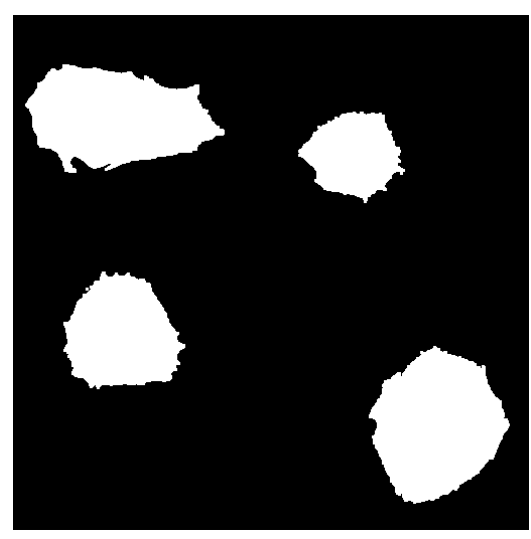

(b)

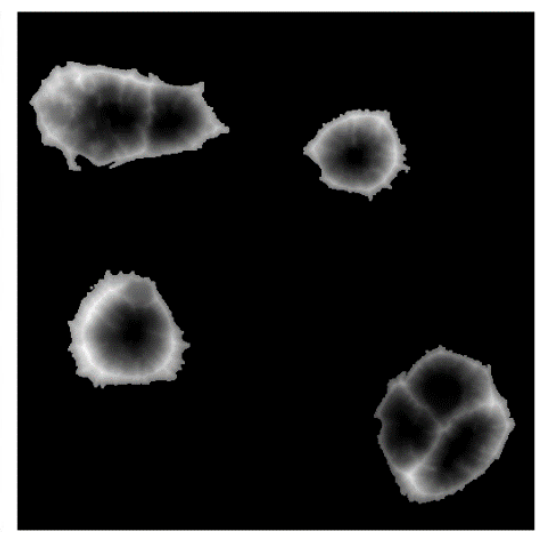

(e)

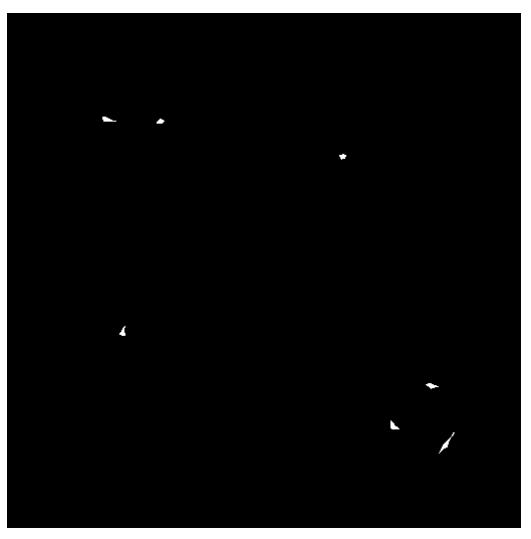

(c)

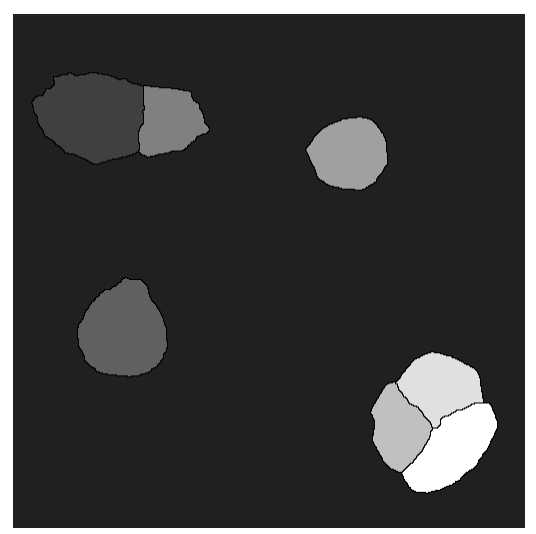

(f)

Figure 4. The steps of cytoplasm segmentation. (a) cytoplasm image after preprocessing and median filtering, (b) filled the whole cytoplasmic area, (c) imposing the nuclei seed as a mask using extendedminima transform, which is the regional minima of the $h$-minima transform level 5, (d) gray-weighted distance transform (GWDT) of the grayscale cytoplasm where the ask is true seed locations, (e) the GWDT applied into - Inf (infinity) background, (f) labeled object after applying watershed segmentation.

\subsection{Post-processing and ruffling area extraction}

For avoiding over- and under-segmentation, we used region merging method. The post-processing algorithm took a final segmented-labeled image, calculated the number of watershed flooded areas and retrieved them as sub-images with real intensity from the real image. An intensity pixel list was created to put the intensity values of labeled regions. If it found any small object, then it compared its intensity value with neighboring regions. If it found any single touching object then the label was put into a merging list. In the case of several touching objects, it took the highest summed intensity of touching objects to the merging list. When the merging list was ready, then objects were merged according to the merging list. Zero in the merging list represented objects that should be discarded as noise. The flowchart of the post-processing is shown in Figure 5. 


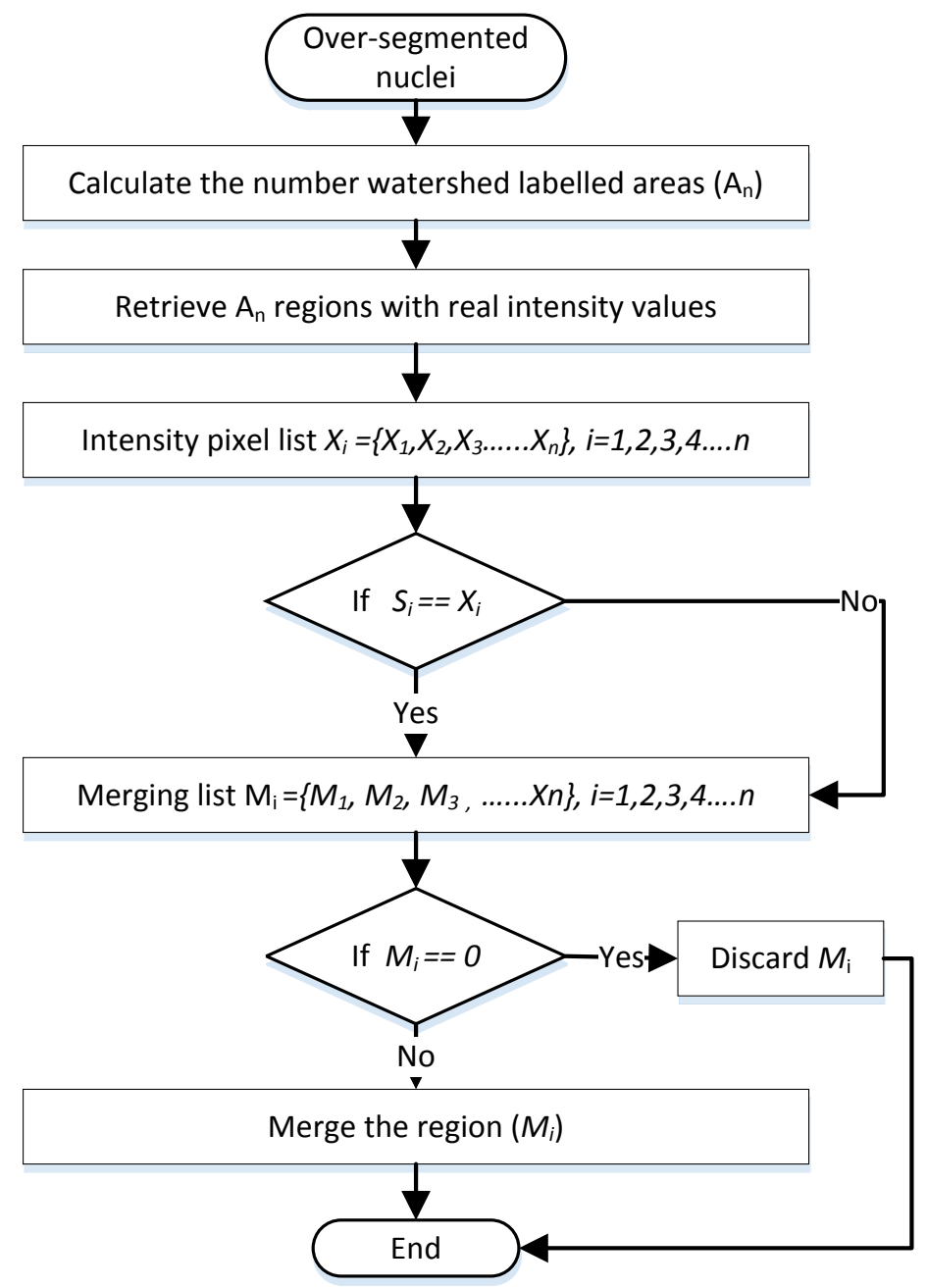

Figure 5. The flows chat of post-processing that used during the marker control watershed segmentation process.

\subsection{Gray-level histograms (GLHs)}

The gray-scale histogram of an image presents the compilation of grayscale value of each pixel in the image. It is presented as if each pixel is placed in a bin corresponding to the color intensity of that pixel. Then all the bins are visualized as a histogram where frequencies of different intensity levels can be seen and different quantitative measurements can be obtained based on this. The grayscale value of the pixel is within the range of $0-255$; the minimum 0 states the pixel is black and 255 indicates the pixel as pure white. Let, $I$ is a gray-scale given image and the intensity histogram is defined by a probability density function $p(k)$ as follows

$$
p(k)=\frac{h(k)}{M}, \quad 0 \leq k \leq n-1
$$

Where $\mathrm{h}(\mathrm{k})$ is the number of pixels in the image or region of interest and $\mathrm{k}$ is the number of grayscale layer and $\mathrm{M}$ is the total number pixels in the image (image height $\times$ image width) 
The histogram bins can provide the following statistical features

Mean: the mean grayscale value of the converted grayscale image

$$
\operatorname{Mean}(\mu)=\sum_{k=0}^{n-1} k p(k)
$$

Variance: variability of discrete measurement of the grayscale value while compared with the mean

$$
\text { Variance }=\sigma^{2}=\sum_{k=0}^{n-1}(k-\mu)^{2} p(k)
$$

Skewness: describes the degree of asymmetry in grayscale values in the image when compared with the mean distribution. Skewness can be positive and negative which reflect right and left asymmetry respectively:

$$
\text { Skewness }=\frac{1}{\sigma^{3}} \sum_{k=0}^{n-1}(k-\mu)^{3} p(k)
$$

Kurtosis: reflects whether the image's grayscale values are heavy-tailed or light-tailed relative to the mean value or not.

$$
\text { Kurtosis }=\frac{1}{\sigma^{4}} \sum_{k=0}^{n-1}(k-\mu)^{4} p(k)
$$

\subsection{Grey-level co-occurrence matrix (GLCM)}

The GLCM algorithm was first introduced by Haralick et al., (1973) and it was built on the conditions of probability density function of two pixels in a certain direction and distance. Basically, it is statistical approach of texture analysis which estimates the second-order joint probability $P_{\delta}(i, j)$ of the intensity values of two pixels ( $i$ and $j$ ) where $\delta$ is a distance apart along a given direction $\theta$. In this paper, extracted ruffling image regions were 8 -bit which gave 256 rows $\times 256$ columns GLCM dimensions for a given displacement vector. We calculated GLCM features for each ruffling image sample for all directional angles $\left(0,45,90\right.$ and $\left.135^{\circ}\right)$. We set $\delta=4$ and then calculated five texture descriptors. Let $P_{\delta}(i, j)$ be the $(i, j)$ th entry in a normalized GLCM. The mean and standard deviations for the rows and columns of the matrix are

$$
\mu_{x}=\sum_{i=1}^{n} i \sum_{j=1}^{n} P_{\delta}(i, j)
$$




$$
\begin{gathered}
\mu_{y}=\sum_{j=1}^{n} i \sum_{i=1}^{n} P_{\delta}(i, j) \\
\sigma_{x}=\sum_{i=1}^{n}\left(i-\mu_{x}\right)^{2} \sum_{j=1}^{n} P_{\delta}(i, j), \\
\sigma_{y}=\sum_{j=1}^{n}\left(j-\mu_{y}\right)^{2} \sum_{i=1}^{n} P_{\delta}(i, j)
\end{gathered}
$$

The descriptors are as follows

(1) Angular second moment (ASM)

$$
A S M=\sum_{i=1}^{n} \sum_{j=1}^{n}\left\{P_{\delta}(i, j)\right\}^{2}
$$

(2) Entropy (ENT)

$$
E N T=\sum_{i=1}^{n} \sum_{j=1}^{n} P_{\delta}(i, j) \times \log \left(P_{\delta}(i, j)\right)
$$

(3) Inverse different moment (IDM)

$$
I D M=\sum_{i=1}^{n} \sum_{j=1}^{n} \frac{1}{1+(i-j)^{2}} P_{\delta}(i, j)
$$

(4) Correlation (COR)

$$
\operatorname{COR}=\left\{\sum_{i=1}^{n} \sum_{j=1}^{n} P_{\delta}(i, j)-\mu_{x} \mu_{y}\right\} / \sigma_{x} \sigma_{y}
$$

(5) Contrast (CON)

$$
\text { Constrast }=\sum_{l=0}^{n-1} l^{2}\left\{\sum_{i=1}^{n} \sum_{j=1}^{n} P_{\delta}(i, j)\right\}, \quad|i-k|=l
$$

\section{Results}

A total of 168 confocal image samples were supplied to the proposed algorithm where 496 cells were manually annotated. The number of cells was grown on different ECM substrate were plastic (107), fibronectin (121), laminin (123) and collagen I (145). The methods were coded in MATALB (R2014a) in a custom way. ImageJ LOCI toolbox was used to subsample the raw images by a factor 2 
to speed up the processing before supplying to the MATLAB custom code (Schneider et al., 2012). The experiments were conducted using a machine with an Intel(R) Xeon(R) CPU E5-2650 v3@2.3GHz, 20 Cores Server, (25 MB Smart Cache) and 64 GB of RAM. Statistical analysis was carried out with Minitab and Microsoft Excel. Data are presented as mean \pm standard error of the mean. Significance was judged using Student's t-test and defined $\mathrm{p}<0.05$.

\subsection{Parameter setting and segmentation results}

The proposed model has two segmentation procedures and the performance of cytoplasm segmentation was depended on the success of nuclei segmentation, as nuclei were used as seeds for cytoplasm segmentation. Throughout the segmentation process, some parameters were tuned to produce good segmentation performance. Parameters used in two segmentation procedures are summarized in Tables 1.

Table 1. Parameters values used in nuclei and cytoplasm binarization steps.

\begin{tabular}{ll}
\hline Used parameters & Values \\
\hline Nuclei segmentation & Sobel \\
MATLAB 2-D filter & 50 pixels \\
Removed object size during morphological opening & 400 pixels \\
Remove particle size after morphological operation & 2 \\
H-minima level & \\
Cytoplasm segmentation & Sobel \\
MATLAB 2-D filter & Line shaped, 2 pixel \\
Morphological opening & 100 pixels \\
Removed object size during morphological opening & 5 \\
h-minima level & Quasi-Euclidian \\
GWDT chamfer weights method &
\end{tabular}

Abbreviations: GWDT-gray weighted distance transform

Both nuclei and cytoplasm segmentation algorithms used subsampled images as input and a median filter were used with a value of $(3,3)$ in the neighborhood around the corresponding pixel in the input image. During nuclei segmentation, a mathematical morphological operation was implemented where the threshold image was dilated. After the morphological operation, EDT was applied and a mask was made from the output of distance transform (DT) by applying $h$-minima transform, which made a 
good mask for identifying the position of nuclei. Then, the DT map and mask were superimposed to get a good mask before applying the watershed algorithm. The watershed was applied, as watershed successfully finds the basin of the nuclei. Once the method had the nuclei seeds, the cytoplasm segmentation procedure took seeded nuclei to find the accurate position of the cytoplasm by using extended h-minima transform. After that, the GWDT was applied with the mask of seeded nuclei preceded by applying a median filter to the cytoplasm. The method superimposed the GWDT map to the mask, which revealed the watershed catchment basin lines. Then, the watershed algorithm was applied and a boundary was drawn. In the case of under- or over-segmentation, those cells were supplied to a post-processing algorithm to allow merging the relevant regions. After the nuclei and cytoplasm were separated, then the ruffling regions were extracted from the whole cell image sample. The results of whole segmentation process are displayed in Figure 6.

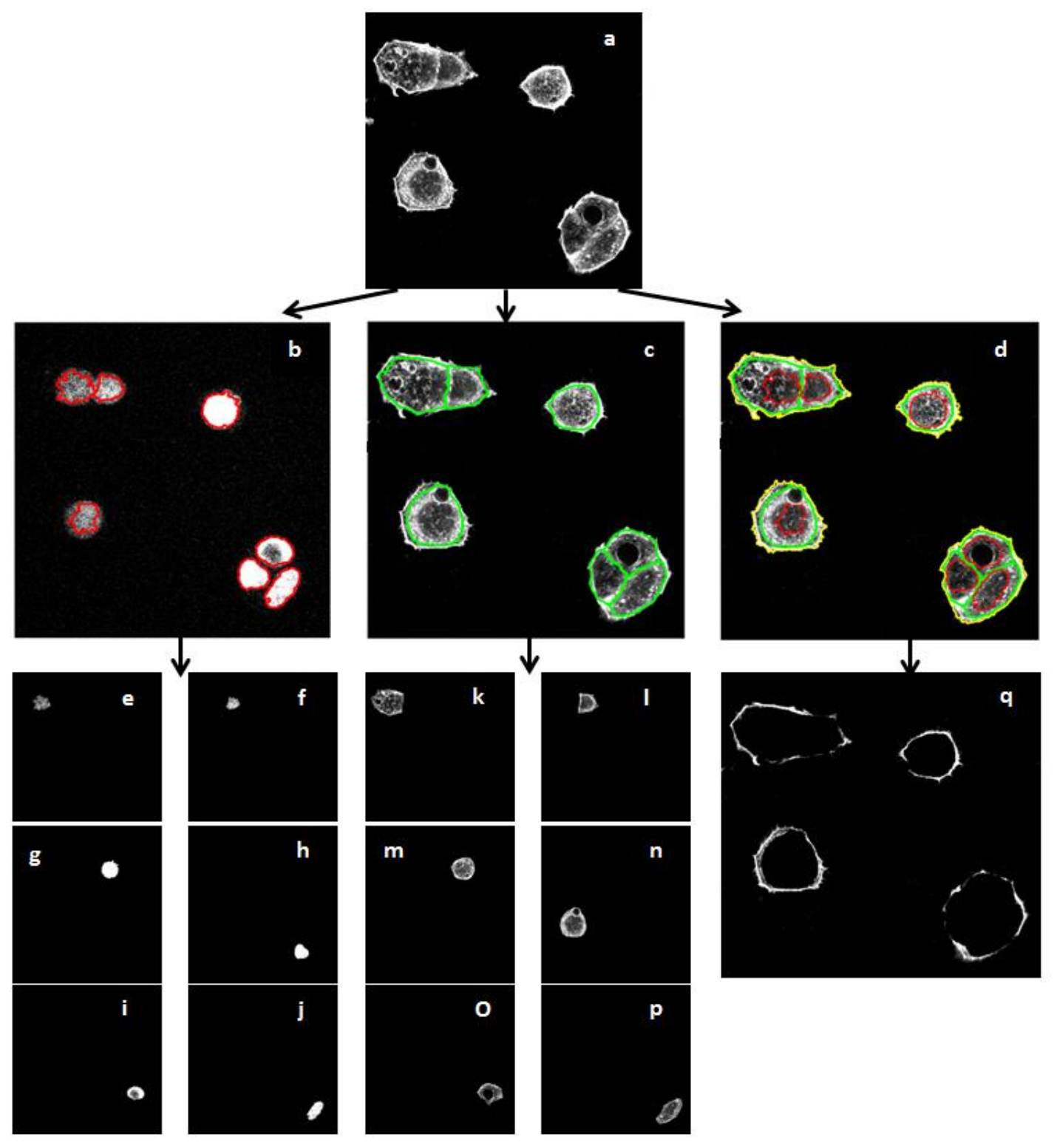


Figure 6. The summary of segmentation results, (a) combined image sample of two channels, (b) the segmented nuclei after nuclei segmentation step, the nuclei are outlined with red color line, (c) the segmented cytoplasm after cytoplasm segmentation step, the cytoplasmic areas are outlined by green color line, (d) ruffling regions are highlighted using yellow line along with nuclei and cytoplasm, (e-f) separated nuclei as single sample, (k-p) separated cytoplasmic areas as single sample, and (q) the identified ruffling regions separated from whole cell image.

\subsection{Analysis of GLH features}

The means and standard deviations of gray-level histogram parameter values were calculated for separated ruffling regions to analyze the complexity of the grayscale distribution. The results of GLH parameters are presented in Table 2. Mean and variances of control and maspin do not carry much information, but skewness and kurtosis values indicate that plastic cells with maspin has the highest skewness and kurtosis value while it is also showing high energy and low entropy value (data not shown). It clearly says that while maspin was present in the cells, the grayscale parameters are uniformly distributed. But for plastic, the values of skewness and kurtosis are highest in compared with others. The lower value of kurtosis for control images show that GLH distributions were dominated my extreme values that maspin images.

Table 2. Characteristics of gray-level histogram parameter values of ruffling regions for four different extracellular matrix (ECM) substrates (plastic, collagen I, laminin, and fibronectin) on control (no maspin) and maspin (with maspin) experimental images ${ }^{\mathrm{a}}$

\begin{tabular}{|l|l|l|l|l|l|l|l|l|}
\hline GLH & \multicolumn{2}{|c|}{ Plastic $(\mathrm{n}=43)$} & \multicolumn{2}{c|}{ Collagen I $(\mathrm{n}=30)$} & \multicolumn{2}{c|}{ Laminin $(\mathrm{n}=46)$} & \multicolumn{2}{c|}{ Fibronectin $(\mathrm{n}=49)$} \\
\hline \multirow{2}{*}{ Mean } & control & maspin & control & maspin & control & maspin & \multicolumn{1}{c|}{ control } & maspin \\
& 1.24 & 1.23 & 1.20 & $1.23(0.1)$ & 1.48 & 1.39 & 1.37 & 1.26 \\
& $(0.09)$ & $(0.09)$ & $(0.07)$ & & $(0.27)$ & $(0.19)$ & $(0.13)$ & $(0.09)$ \\
\hline Varia & 0.70 & 1.04 & 0.77 & 0.72 & 2.29 & 1.82 & 1.67 & 1.12 \\
nce & $(0.49)$ & $(0.42)$ & $(0.42)$ & $(0.55)$ & $(1.42)$ & $(0.95)$ & $(0.75)$ & $(0.47)$ \\
\hline Skew & 4.39 & 5.34 & 5.09 & 4.23 & 3.72 & 3.99 & 3.96 & 4.63 \\
ness & $(1.04)$ & $(1.25)$ & $(0.79)$ & $(0.89)$ & $(1.30)$ & $(1.09)$ & $(0.89)$ & $(0.79)$ \\
\hline Kurto & $\mathbf{2 6 . 9 8}$ & $\mathbf{3 3 . 7 3}$ & $\mathbf{3 1 . 5 8}$ & $\mathbf{2 3 . 7 0}$ & $\mathbf{1 8 . 3 8}$ & $\mathbf{2 0 . 3 6}$ & $\mathbf{1 9 . 3 7}$ & $\mathbf{2 5 . 7 3}$ \\
sis & $\mathbf{( 1 3 . 6 3 )}$ & $\mathbf{( 1 4 . 0 5 )}$ & $\mathbf{( 9 . 2 9 )}$ & $\mathbf{( 9 . 5 4 )}$ & $\mathbf{( 1 3 . 2 8 )}$ & $\mathbf{( 1 2 . 8 9 )}$ & $\mathbf{( 8 . 4 7 )}$ & $\mathbf{( 9 . 1 8 )}$ \\
\hline
\end{tabular}

${ }^{a}$ All values are presented as mean \pm SD from number of image samples (n)

\subsection{Analysis of GLCM features}

Figure 7 presents the GLCM parameters comparison between control and maspin using student t-test. It is plausible to mention that control cells are showing more polarized characteristics as they have high ASM, CON, COR and IDM values and low ENT values. But on the other hand, maspin cells 
show more regular and non-polarized characteristics. It is evident from the correlation values that maspin cells are more compact as they have lower COR values. From visual inspection, we also confirmed that there was dense meshwork of cellular actin at the periphery. Similar types of measurements have been found in Lan et al., (2015) where microtubules patterns were quantified using GLCM parameters.

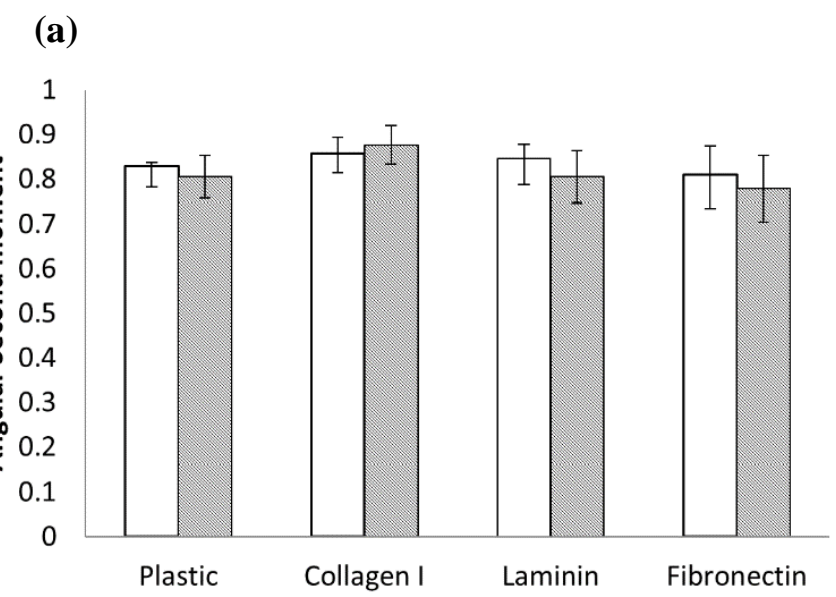

(b)

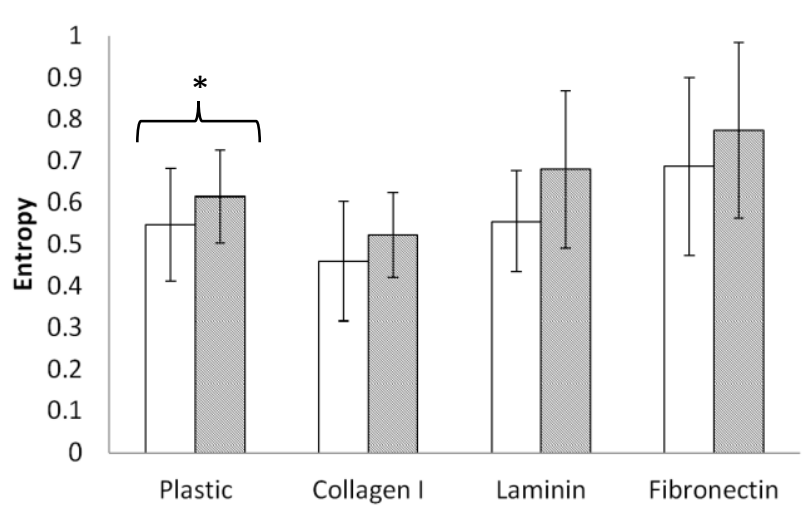

(d)

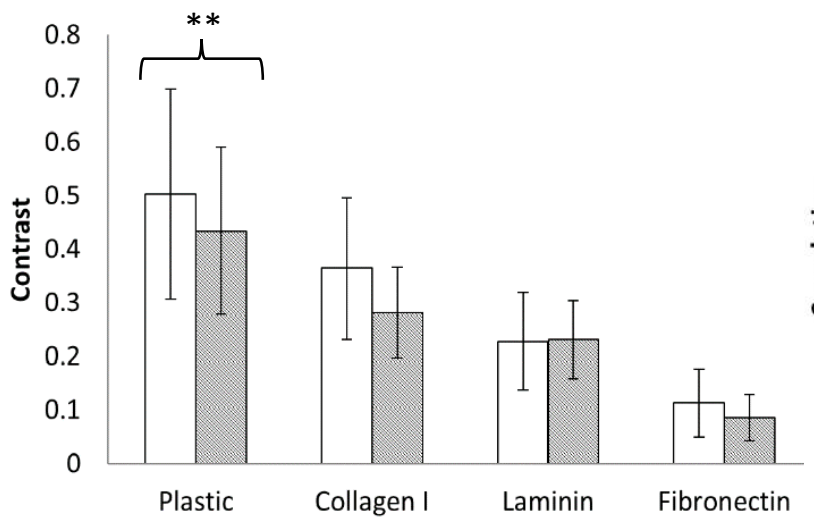

(c)

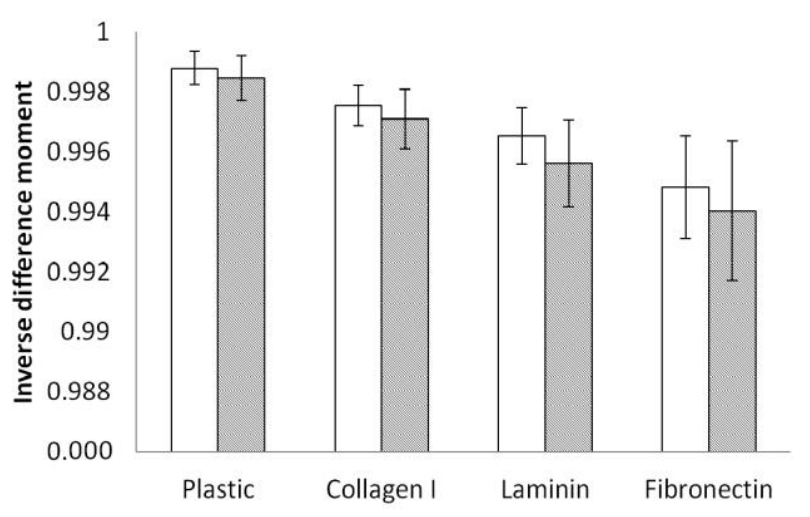

(e)

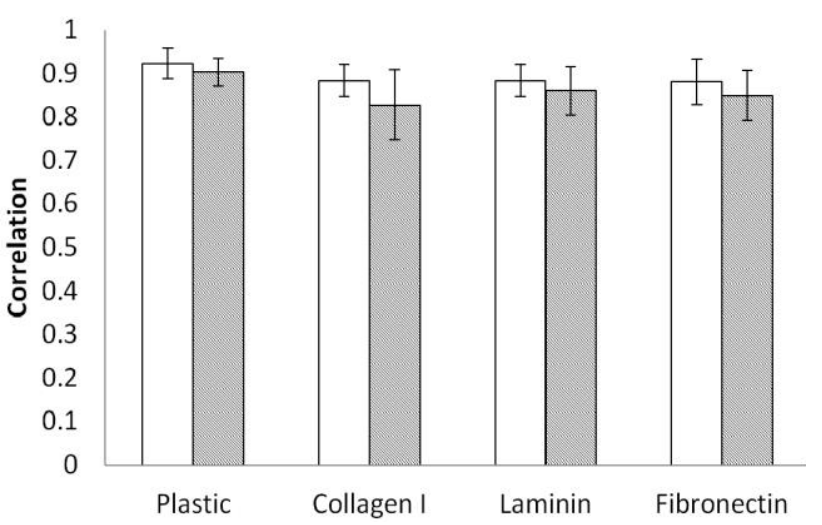

Figure 7. Texture analysis results of selected gray-level co-occurrence matrix (GLCM) parameters: (a) energy, (b) entropy, (c) inverse difference moment (IDM), and (e) contrast for maspin exposure for four different extracellular matrix (ECM) substrates (plastic, collagen I, laminin, and fibronectin). 
White bars represent control experiments while colored bars present the maspin experiments. *,p $<0.01 ; * * p<0.005$.

\subsection{Maspin alters cellular concentrations of $G$ and $F$ actin}

The influence of maspin on the cellular levels of globular and filamentous actin was investigated, as this can be related to cell motility. Biochemical fractionation was used to separate soluble G-actin and insoluble F-actin from MCF7 cells stably expressing pcDNA-3.2 or pcDNA3.2-Maspin, that were plated on plastic, collagen I, laminin or fibronectin (Figure 8). MCF7-pcDNA-Maspin cells were found to have increased levels of F-actin in comparison to MCF-7-pcDNA-3.2. The greatest increase (34\%) was observed in cells grown on plastic or collagen I. MCF-7-pcDNA3.2-Maspin cells grown on plastic, collagen I and laminin showed an increase in G-actin content in comparison to control cells. All cells grown on fibronectin showed equal expression of G-actin. Generally, maspin caused an increase in the cellular content of both G-actin and F-actin.

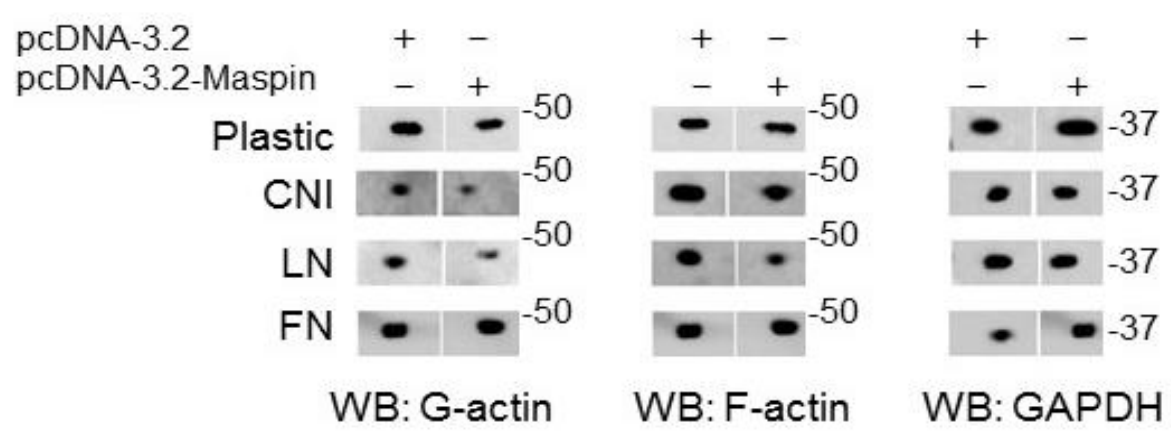

Figure 8: Biochemical fractionation of actin in MCF-7 cells. MCF-7 stably transfected with pcDNA3.2 or pcDNA3.2-Maspin were grown on isolated ECM components: plastic, collagen I (CNI), laminin (LN), or fibronectin (FN). Cell lysates were separated into soluble (containing G-actin) and insoluble (containing F-actin) fractions. $10 \mu \mathrm{g}$ of the soluble fraction and $30 \mu \mathrm{g}$ of the insoluble fraction were separated by SDS-PAGE and detected on western blots using anti-actin $(200 \mathrm{ng} / \mathrm{ml})$ or anti-GAPDH (1:5000). Staining performed on three independent samples, representative images selected.

\subsection{Evaluation of segmentation algorithms}

To avoid the over-segmentation in cytoplasm it was necessary to provide perfect nuclei seed to the cytoplasm segmentation procedures. Both procedures passed the under- or over-segmented images to the post-processing algorithm (shown in figure 9a). When the algorithm got the final segmented labelled image (shown in figure $9 \mathrm{~b}$ ), it calculated the number of watershed flooded area and retrieved them as sub-images with real intensity from the real image (shown in figure $9 \mathrm{c}$ ). Then the method got 
the final segmented image, which was confirmed by human annotation and evaluation (shown in Figure 9.d).

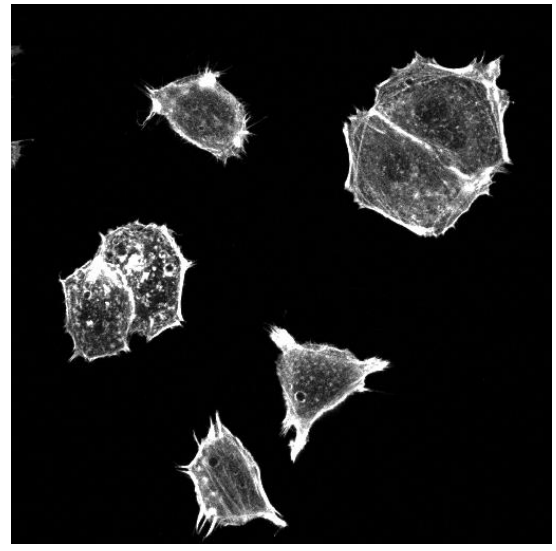

(a)

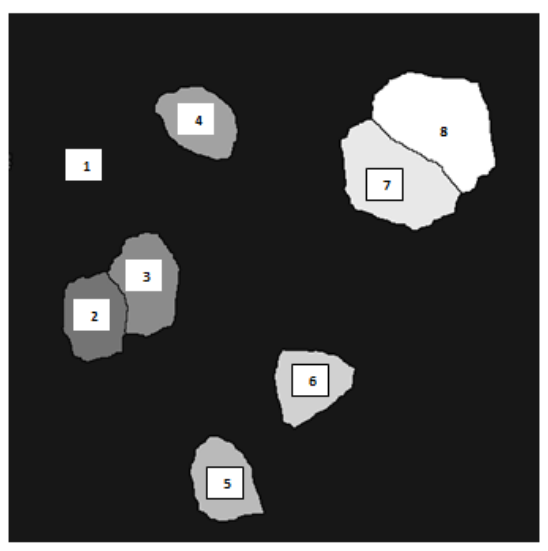

(c)

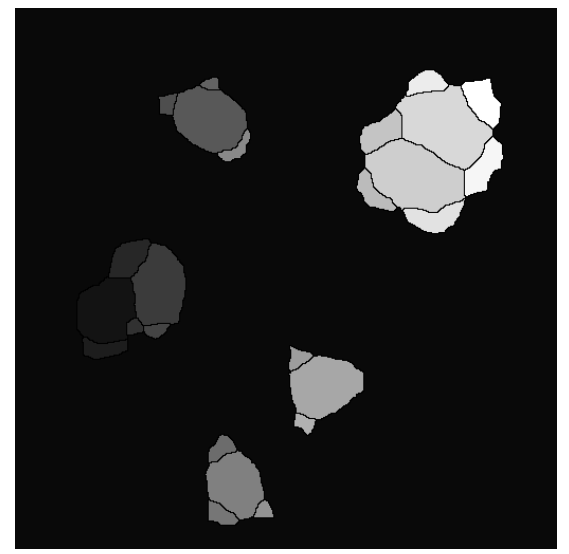

(b)

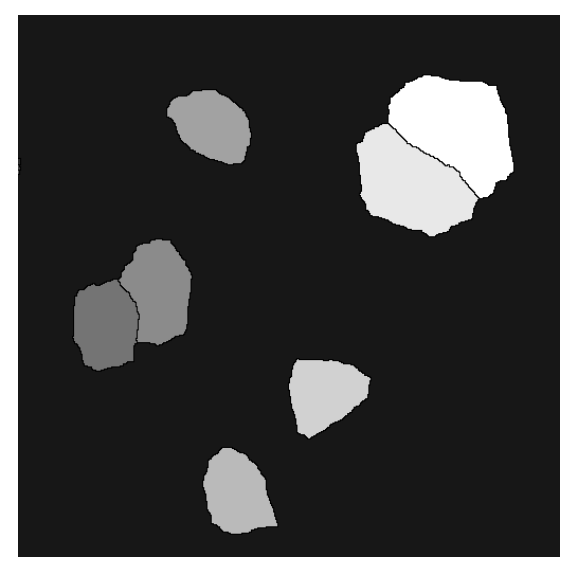

(d)

Figure 9: The post-processing method for proposed marker-controlled watershed segmentation (MCWS) method, a) the filtered grayscale image, b) the over-segmented cytoplasm, c) identified regions during the post-processing method based on which area, and d) final segmented cytoplasm after applying the post-processing algorithm.

\section{Discussion}

It has been reported previously that maspin triggers several cellular processes which get reflected on cell behavior and cytoskeletal architecture (Odero-Marah et al., 2003; Qin \& Zhang, 2010; Lara et al., 2012; Al-Mamun et al., 2016a). In a recent attempt, Al-Mamun et al., (2016b) showed that an image processing tool can be used to quantify three cellular parts: nuclei, cytoplasm and ruffling regions to observe the changes in shape and behavior. The study considers descriptors including area, perimeter, integrated optical density, fractal dimension and circularity to quantify each cellular part. In this 
paper, we focused on the cellular periphery machinery and built a quantitative method that combines the image segmentation and two texture analysis methods to quantify the ruffling area textures. GLH and GLCM texture parameters were calculated and analyzed in Figure 7. A biochemical fractionation experiment was used to separate soluble G-actin and insoluble F-actin on four different ECM substrates for stably expressing pcDNA-3.2, pcDNA3.2-Maspin. Recently, Endsley et al., (2011) suggests that two pathways that how maspin enhances cell-ECM adhesion while reduces cell migration. First, maspin binds to uPA and localises on the cell surface by mapsin-uPA binding to uPAR. In this case, uPAR and $\beta 1$ integrin do not reside in a complex. Then, the maspin -uPA-uPAR complex associates with and inactivates $\beta 1$ integrin (via the G-helix of maspin binding to $\beta 1$ integrin), thus reducing cell migration potentially by increasing cell adhesion. Second, uPAR and $\beta 1$ integrin are already in complex, thus facilitating reduced migration capabilities. The second possibility, which is probably more prevalent in cancer cells, involves uPAR and $\beta 1$ integrin already in complex, thus facilitating enhanced migration capabilities. Then, the maspin-uPA complex binds to (or associates with) the uPAR- $\beta 1$ integrin complex, thereby inducing a conformational or lateral mobility change in uPAR, which causes an altered physical association (and/or activation state) with $\beta 1$ integrin, eventually resulting in decreased cell migration and/or increased cell adhesion.

Looking at the texture parameters value we found a common ground to show that cells without having maspin are non-polarized. This phenomenon is also confirmed by biochemical fractionation where we saw that maspin cells grown on plastic, collagen I and laminin showed an increase in G-actin content in comparison to the other cell types. Also, cells transfected with wild type maspin showed a slight increase in the content of both G-actin and F-actin which corresponds with its phenotype of a thick actin periphery and large flattened phenotype. It supports the finding that MCF7 cells stably expressing wild type maspin significantly increased cell adhesion by $113 \pm 5 \%$ on a laminin matrix and by $4576 \%$ on either collagen I or fibronectin matrices, in comparison to cells expressing vector only (Al-Mamun et al., 2016a). Also, GLCM values from our current analysis, we saw that ruffling regions of control cells (without maspin) are polarized and elongated as their entropy value is lower.

To evaluate the segmentation method, we compared our segmentation results with the level set method. The level set method is a popular and established numeric method for segmenting shapes and contours (Osher \& Fedkiw, 2003, Li, et al., 2010). For keeping the experiments simple, we replaced the binarization by the level set method proposed by Li et al., (2010). Specifically, the method adopted Distance Regularized Level Set Method (DRLSE) algorithm where several parameter $(\lambda, \mu, \Delta t, \alpha)$ values were used. We ran the algorithms with four parameters and fixed the values by an iterative process. $\lambda=5, \mu=0.02, \Delta t=5.0$ and $\alpha=1.5$. It has been discussed in LaTore et al., (2013) that large values of $\alpha$ may lead to boundary leakage, in our experiments we choose low alpha values in a range $(0.5-2.5)$. Moreover, we used DT map for nuclei segmentation while we used DT and GWDT both for cytoplasm segmentation for validation purpose. Table 3 summarizes the average 
segmentation accuracy for three cell parts using without GWDT, with GWDT and level set when compared with manually detected cells. When there is an error or over-segmentation occurred, it means the error occurred in at least one part of segmentation. To presents the accuracy we divided it into different error. Type-I error refers to cells had faced over-segmentation (they have been divided into multiple parts when they should have not). Type-II error occurs when the algorithm detected background noise as a cell in the binarization process. Finally, Type-III error represents the image samples which were rejected because of intensity problem.

Table 3. Validation of segmentation accuracy and comparison of three methods used during binarization process: a model without gray-weighted distance transform (GWDT), a model with GWDT and level set method.

\begin{tabular}{|c|c|c|c|}
\hline & $\begin{array}{l}\text { Model without } \\
\text { GWDT }\end{array}$ & Model with GWDT & Level set \\
\hline Number of manually detected cells & 496 & 496 & 496 \\
\hline Correctly Segmented (\%) & 81.05 & 92.54 & 91.54 \\
\hline Type-I error: Over-segmentation (\%) & 11.50 & 2.22 & 3.13 \\
\hline Type-II: Noise detected as cells (\%) & 2.62 & 0.8 & 0.8 \\
\hline Type-III error: intensity problem (\%) & 4.83 & 4.43 & 4.43 \\
\hline
\end{tabular}

Table 3 indicates that model without using GWDT in cytoplasm segmentation gives the lowest accuracy, over-segmentation could not be avoided, but a model with GWDT performs slightly better than level set method. But when we tuned the parameters in the level set method, it was seen that the method was really sensitive to the image sample that had intensity problems and that results in a slightly higher percentage of over-segmentation.

Researchers have been trying to investigate subcellular cell images to extract both qualitative and descriptive information from nuclei, cytoplasm, and other parts. But clumped nuclei and cytoplasm, uneven illumination of images and subject specific limitations of image tools challenge the quantitative image analysis tool. In this paper, we developed an image segmentation tool using marker control watershed segmentation and further integrated texture analysis method to analyze the cellular cytoskeleton of the cells. We developed several in vitro experiments of tumor cell line MCF-7 with different maspin exposure on different ECM substrates (plastic, collagen I, laminin and fibronectin) to support our computational evidence. However, this paper provides a quantitative image analysis method that can segment nuclei, cytoplasm and ruffling regions of single and as well as clumped cells, while the segmentation results were validated using domain expert's knowledge. The method integrated two texture analysis methods: GLH and GLCM to find some important information about the shape and behavior of the ruffling region. 
This paper differs from the previous model developed by Al-Mamun et al., (2016b) in various ways. First, the current version of the method is compared with the level set method suggested in Li et al., (2010), but no comparison was attempted in Al-Mamun et al., (2016b). But it is needed to mention that level set method performs almost similar to the current method. Second, we included an explicit texture analysis method (both GLH and GLCM) that confirmed the specific changes of ruffling regions due to maspin. Third, we provided in vitro support to our computational hypothesis that maspin acts as cellular cytoskeleton regulator. But our model has some limitations. Our current model did not consider the precise measurements of filopodia, lammellepodia, stress fiber, and focal adhesions. Also, it could not provide cell-ECM adhesion measurement due to lack of data collection. In future, the addition of these measurements can provide more evidence that maspin enhances cellECM adhesion by reducing EMT process while reduces cell migration in a subsequent process.

\section{Conclusion}

In conclusion, the present work has combined image segmentation and texture analysis techniques to quantify the cellular cytoskeletal changes in terms of shape and behavior. We demonstrate that reduction of cell migration effects of maspin can be facilitated by cellular cytoskeletal changes due to maspin presence. Overall, this tool can be used by biologist, cancer specialist and computer scientists to perform similar types of quantification studies on subcellular complex images.

\section{Reference}

Al-Mamun, M. A., Brown, L. J., Hossain, M. A., Fall, C., Wagstaff, L., \& Bass, R. (2013). A hybrid computational model for the effects of maspin on cancer cell dynamics. Journal of theoretical biology, 337, 150-160.

Al-Mamun, M. A., Farid, D. M., Ravenhil, L., Hossain, M. A., Fall, C., \& Bass, R. (2016a). An in silico model to demonstrate the effects of Maspin on cancer cell dynamics. Journal of theoretical biology, 388, 37-49.

Al-Mamun, M., Ravenhill, L., Srisukkham, W., Hossain, A., Fall, C., Ellis, V., \& Bass, R. (2016b). Effects of Noninhibitory Serpin Maspin on the Actin Cytoskeleton: A Quantitative Image Modeling Approach. Microscopy and microanalysis: the official journal of Microscopy Society of America, Microbeam Analysis Society, Microscopical Society of Canada, 22(2), 394-409.

Amini, S., Veilleux, D., \& Villemure, I. (2010). Tissue and cellular morphological changes in growth plate explants under compression. Journal of biomechanics, 43(13), 2582-2588.

Arco, J. E., Górriz, J. M., Ramírez, J., Álvarez, I., \& Puntonet, C. G. (2015). Digital image analysis for automatic enumeration of malaria parasites using morphological operations. Expert Systems with Applications, 42(6), 3041-3047.

Bass R, Wagstaff L, Ravenhill L \& Ellis V. (2009) Binding of extracellular maspin to beta1 integrins inhibits vascular smooth muscle cell migration. Journal of Biological Chemistry 284(40), 27712-20.

Breu, H., Gil, J., Kirkpatrick, D., \& Werman, M. (1995). Linear time Euclidean distance transform algorithms. IEEE Transactions on Pattern Analysis and Machine Intelligence, 17(5), 529-533.

Cai, H., Yang, Z., Cao, X., Xia, W., \& Xu, X. (2014). A new iterative triclass thresholding technique in image segmentation. IEEE transactions on image processing, 23(3), 1038-1046.

Cella N, Contreras A, Latha K, Rosen JM, \& Zhang M. (2006). Maspin is physically associated with (beta)1 integrin regulating cell adhesion in mammary epithelial cells. FASEB Journal 20(9), 1510-2. 
Chakrabarti, K. R., Hessler, L., Bhandary, L., \& Martin, S. S. (2015). Molecular Pathways: New Signaling Considerations When Targeting Cytoskeletal Balance to Reduce Tumor Growth. Clinical Cancer Research, 21(23), 5209-5214.

Dang, Y., Lan, X., Zhang, Q., Li, L., \& Huang, Y. (2015). Analysis of grayscale characteristics in images of labeled microtubules from cultured cardiac myocytes. Microscopy and Microanalysis, 21(02), 334-342.

Debeir, O., Van Ham, P., Kiss, R., \& Decaestecker, C. (2005). Tracking of migrating cells under phase-contrast video microscopy with combined mean-shift processes. IEEE transactions on medical imaging, 24(6), 697-711.

Endsley, M. P., Hu, Y., Deng, Y., He, X., Warejcka, D. J., Twining, S. S., ... \& Zhang, M. (2011). Maspin, the molecular bridge between the plasminogen activator system and $\beta 1$ integrin that facilitates cell adhesion. Journal of Biological Chemistry, 286(28), 24599-24607.

Fenistein, D., Lenseigne, B., Christophe, T., Brodin, P., \& Genovesio, A. (2008). A fast, fully automated cell segmentation algorithm for high-throughput and high-content screening. Cytometry part A, 73(10), 958-964.

Haralick, R. M., \& Shanmugam, K. (1973). Textural features for image classification. IEEE Transactions on systems, man, and cybernetics, (6), 610-621.

Kayser, K., Schultz, H., Goldmann, T., Görtler, J., Kayser, G., \& Vollmer, E. (2009). Theory of sampling and its application in tissue based diagnosis. Diagnostic Pathology, 4(1), 6.

Khalkhali-Ellis, Z., \& Hendrix, M. J. (2007). Elucidating the Function of Secreted Maspin: Inhibiting Cathepsin D-Mediated Matrix Degradation. Cancer research, 67(8), 3535-3539.

Koyuncu, C. F., Arslan, S., Durmaz, I., Cetin-Atalay, R., \& Gunduz-Demir, C. (2012). Smart markers for watershed-based cell segmentation. PloS one, 7(11), e48664.

Lan, X., Li, L., Hu, J., Zhang, Q., Dang, Y., \& Huang, Y. (2015). A Quantitative Method for Microtubule Analysis in Fluorescence Images. Microscopy and Microanalysis, 21(06), 1582-1590.

Lara H, Wang Y, Beltran AS, Juárez-Moreno K, Yuan X, Kato S, Leisewitz AV, Cuello Fredes M, Licea AF, Connolly DC, Huang L, \& Blancafort P. (2012). Targeting serous epithelial ovarian cancer with designer zinc finger transcription factors. Journal of Biological Chemistry 287(35), 29873-86.

LaTorre, A., Alonso-Nanclares, L., Muelas, S., Peña, J. M., \& DeFelipe, J. (2013). Segmentation of neuronal nuclei based on clump splitting and a two-step binarization of images. Expert Systems with Applications, 40(16), 6521-6530.

Law, R. H., Irving, J. A., Buckle, A. M., Ruzyla, K., Buzza, M., Bashtannyk-Puhalovich, T. A., ... \& Bird, P. I. (2005). The high resolution crystal structure of the human tumor suppressor maspin reveals a novel conformational switch in the G-helix. Journal of Biological Chemistry, 280(23), 22356-22364.

Li, C., Xu, C., Gui, C., \& Fox, M. D. (2010). Distance regularized level set evolution and its application to image segmentation. IEEE transactions on image processing, 19(12), 3243-3254.

Lindblad, J., Wählby, C., Bengtsson, E., \& Zaltsman, A. (2004). Image analysis for automatic segmentation of cytoplasms and classification of Rac1 activation. Cytometry Part A, 57(1), 22-33.

Lu, Y., Huang, C., Wang, J., \& Shang, P. (2014). An improved quantitative analysis method for plant cortical microtubules. The Scientific World Journal, 2014.

Matuszewski, B. J., Murphy, M. F., Burton, D. R., Marchant, T. E., Moore, C. J., Histace, A., \& Precioso, F. (2011, September). Segmentation of cellular structures in actin tagged fluorescence confocal microscopy images. In 2011 18th IEEE International Conference on Image Processing (pp. 3081-3084). IEEE.

Mukherjee, D. P., Ray, N., \& Acton, S. T. (2004). Level set analysis for leukocyte detection and tracking. IEEE Transactions on Image processing, 13(4), 562-572.

Nath, S. K., Palaniappan, K., \& Bunyak, F. (2006, October). Cell segmentation using coupled level sets and graph-vertex coloring. In International Conference on Medical Image Computing and Computer-Assisted Intervention (pp. 101-108). Springer Berlin Heidelberg. 
Ngamkitidechakul, C., Burke, J. M., O’Brien, W. J., \& Twining, S. S. (2001). Maspin: synthesis by human cornea and regulation of in vitro stromal cell adhesion to extracellular matrix. Investigative ophthalmology \& visual science, 42(13), 3135-3141.

Ngamkitidechakul, C., Warejcka, D. J., Burke, J. M., O'Brien, W. J., \& Twining, S. S. (2003). Sufficiency of the Reactive Site Loop of Maspin for Induction of Cell-Matrix Adhesion and Inhibition of Cell Invasion Conversion of Ovalbumin to a Maspin-like Molecule. Journal of Biological Chemistry, 278(34), 31796-31806.

Odero-Marah, V. A., Khalkhali-Ellis, Z., Chunthapong, J., Amir, S., Seftor, R. E., Seftor, E. A., \& Hendrix, M. J. (2003). Maspin regulates different signaling pathways for motility and adhesion in aggressive breast cancer cells. Cancer biology \& therapy, 2(4), 398-403.

Qin L, \& Zhang M. (2010). Maspin regulates endothelial cell adhesion and migration through an integrin signaling pathway. Journal of Biological Chemistry 285(42), 32360-9

Osher, S., \& Fedkiw, R. (2006). Level set methods and dynamic implicit surfaces (Vol. 153). Springer Science \& Business Media.

Otsu, N. (1979). A threshold selection method from gray-level histograms. IEEE Transactions on Systems, Man and Cybernetics, 9, 62-66.

Quelhas, P., Marcuzzo, M., Mendonça, A. M., \& Campilho, A. (2010). Cell nuclei and cytoplasm joint segmentation using the sliding band filter. IEEE Transactions on Medical Imaging, 29(8), 1463-1473.

Pemberton, P. A., Tipton, A. R., Pavloff, N., Smith, J., Erickson, J. R., Mouchabeck, Z. M., \& Kiefer, M. C. (1997). Maspin is an intracellular serpin that partitions into secretory vesicles and is present at the cell surface. Journal of Histochemistry \& Cytochemistry, 45(12), 1697-1706.

Plissiti, M. E., Nikou, C., \& Charchanti, A. (2011). Combining shape, texture and intensity features for cell nuclei extraction in Pap smear images. Pattern Recognition Letters, 32(6), 838-853.

Ravenhill L, Wagstaff L, Edwards DR, Ellis V \& Bass R. (2010) The G-helix of maspin mediates effects on cell migration and adhesion. Journal of Biological Chemistry 285(47), 36285 - 92.

Ridley AJ. (2011). Life at the leading edge._Cell 145(7),1012-22.

Sander, E. E., Jean, P., van Delft, S., van der Kammen, R. A., \& Collard, J. G. (1999). Rac downregulates rho activity reciprocal balance between both gtpases determines cellular morphology and migratory behavior. The Journal of cell biology, 147(5), 1009-1022.

Seftor, R. E., Seftor, E. A., Sheng, S., Pemberton, P. A., Sager, R., \& Hendrix, M. J. (1998). Maspin suppresses the invasive phenotype of human breast carcinoma. Cancer research, 58(24), 5681-5685.

Sheng, S., Carey, J., Seftor, E. A., Dias, L., Hendrix, M. J., \& Sager, R. (1996). Maspin acts at the cell membrane to inhibit invasion and motility of mammary and prostatic cancer cells. Proceedings of the National Academy of Sciences, 93(21), 11669-11674.

Srisukkham W, Lepcha P, Hossain MA, Zhang L, Jiang R, \& Lim HN. (2013). A Mobile Enabled Intelligent Scheme to Identify Blood Cancer for Remote Areas - Cell Membrane Segmentation using Marker Controlled Watershed Segmentation Phase. In The 7th International Conference on Software, Knowledge, Information Management and Applications (SKIMA), 18-20 Dec, Chiang Mai, Thailand.

Teoh, S. S., Whisstock, J. C., \& Bird, P. I. (2010). Maspin (SERPINB5) is an obligate intracellular serpin. Journal of Biological Chemistry, 285(14), 10862-10869.

Teoh SS, Vieusseux J, Prakash M, Berkowicz S, Luu J, Bird CH, Law RH, Rosado C, Price JT, Whisstock JC, \& Bird PI. (2014). Maspin is not required for embryonic development or tumor suppression. Nature Communications 5, 3164.

Wählby, C., SINTORN, I. M., Erlandsson, F., Borgefors, G., \& Bengtsson, E. (2004). Combining intensity, edge and shape information for $2 \mathrm{D}$ and $3 \mathrm{D}$ segmentation of cell nuclei in tissue sections. Journal of Microscopy, 215(1), 67-76.

Yang, X., Li, H., \& Zhou, X. (2006). Nuclei segmentation using marker-controlled watershed, tracking using mean-shift, and Kalman filter in time-lapse microscopy. IEEE Transactions on Circuits and Systems I: Regular Papers, 53(11), 2405-2414. 
Zou Z, Anisowicz A, Hendrix MJ, Thor A, Neveu M et al. (1994). Maspin, a serpin with tumor-suppressing activity in human mammary epithelial cells. Science 263(5146), 526-529.

Zou, L., Jaramillo, M., Whaley, D., Wells, A., Panchapakesa, V., Das, T., \& Roy, P. (2007). Profilin-1 is a negative regulator of mammary carcinoma aggressiveness. British journal of cancer, 97(10), 1361-1371. 